\title{
Numerical evolutions of spherical Proca stars
}

\author{
Nicolas Sanchis-Gual, ${ }^{1}$ Carlos Herdeiro, ${ }^{2}$ Eugen Radu, ${ }^{2}$ Juan Carlos Degollado, ${ }^{3}$ and José A. Font ${ }^{1,4}$ \\ ${ }^{1}$ Departamento de Astronomía y Astrofísica, Universitat de València, Dr. Moliner 50, \\ 46100 Burjassot (València), Spain \\ ${ }^{2}$ Departamento de Física da Universidade de Aveiro and Centre for Research and Development in \\ Mathematics and Applications (CIDMA), Campus de Santiago, 3810-183 Aveiro, Portugal \\ ${ }^{3}$ Instituto de Ciencias Físicas, Universidad Nacional Autónoma de México, Apdo. Postal 48-3, \\ 62251 Cuernavaca, Morelos, México \\ ${ }^{4}$ Observatori Astronòmic, Universitat de València, C/ Catedrático José Beltrán 2, \\ 46980 Paterna (València), Spain
}

(Received 16 February 2017; published 22 May 2017)

\begin{abstract}
Vector boson stars, or Proca stars, have been recently obtained as fully nonlinear numerical solutions of the Einstein-(complex)-Proca system [1]. These are self-gravitating, everywhere nonsingular, horizonless Bose-Einstein condensates of a massive vector field, which resemble in many ways, but not all, their scalar cousins, the well-known (scalar) boson stars. In this paper we report fully nonlinear numerical evolutions of Proca stars, focusing on the spherically symmetric case, with the goal of assessing their stability and the end point of the evolution of the unstable stars. Previous results from linear perturbation theory indicate that the separation between stable and unstable configurations occurs at the solution with maximal ADM mass. Our simulations confirm this result. Evolving numerically unstable solutions, we find, depending on the sign of the binding energy of the solution and on the perturbation, three different outcomes: (i) migration to the stable branch, (ii) total dispersion of the scalar field, or (iii) collapse to a Schwarzschild black hole. In the latter case, a long-lived Proca field remnant—a Proca wig — composed by quasibound states, may be seen outside the horizon after its formation, with a lifetime that scales inversely with the Proca mass. We comment on the similarities/differences with the scalar case as well as with neutron stars.
\end{abstract}

DOI: 10.1103/PhysRevD.95.104028

\section{INTRODUCTION}

In Minkowski spacetime, the linear, massive, KleinGordon equation has a general solution given in terms of Fourier modes, corresponding to different frequencies. The amplitudes of these modes can be chosen as to yield, at some given time, a lumplike solution, wherein all (or almost all) energy is localized in a compact spatial region. The different phase velocity of the different modes, however, implies this lump will be dispersed by the time evolution.

Solitonlike solutions of the Klein-Gordon field, corresponding to energy lumps that are preserved by the time evolution, require nonlinearities that compensate the field's natural tendency to disperse. These nonlinearities may be due to the field self-interactions [2], or due to gravity. Examples within the latter context were first discovered in $[3,4]$, as static, spherically symmetric solutions of the Einstein-(massive, complex)Klein-Gordon system, and became known as (scalar) boson stars (SBSs) - see [5] for a review.

Unlike the (short-lived) flat space lumps described in the first paragraph, SBSs correspond to a single frequency mode with a very large occupation number. They are sometimes described as relativistic, self-gravitating, Bose-Einstein condensates. This single frequency appears as a harmonic time dependence for the scalar field. Thus, compatibility with a static geometry requires the field to be complex, containing two scalar degrees of freedom, both oscillating but with a phase difference of $\pi / 2$. Physically, these oscillations prevent the collapse of the scalar field into a black hole and simultaneously evade no-soliton Derrick [6]/virial-type theorems. The opposite phases, on the other hand, shut down scalar field dissipation towards infinity, which is observed for real fields, for which only quasistationary configurations exist, albeit extremely long lived [7], dubbed oscillatons [8]. For rotating SBSs $[9,10]$ moreover, the two opposite phases also shut down gravitational wave emission. We remark that SBSs require a mass term but no self-interactions, even though the latter may also be included [11-15].

The existence of boson stars as stationary solutions of the Einstein equations raises questions about their dynamics (see [16] for a review). In particular, one may ask if SBSs are stable, and, even more fundamentally, if they may form dynamically. For spherically symmetric SBSs, the first question was answered at the level of mode stability in $[17,18]$. Roughly, fundamental solutions (i.e. without nodes in the scalar field radial profile) that become too compact are unstable against radial perturbations. More precisely, the turnover point, stability-wise, corresponds to the solution with the maximum ADM mass for a given scalar field mass. These perturbative results were confirmed by fully nonlinear numerical simulations in [19], where, 
moreover, the fate of the unstable solutions was reported to be either the formation of a black hole, or the migration to the stable branch, regardless of the sign of the binding energy. A different possible fate of unstable SBSs with excess (i.e. negative binding) energy is that they disperse entirely, which was reported in [20] for excited states and [21] for fundamental states (see also [22]).

Concerning the second question above, the dynamical formation of SBSs was first examined using nonlinear numerical simulations, in [23], where it was found that stable SBSs can form starting with generic initial data describing an unbound scalar field, after releasing the excess energy via the mechanism of gravitational cooling. For a discussion of SBS formation in cosmological scenarios see [24].

It has long been thought that there could be vector analogues to SBSs, but the former have only recently been constructed and are known as Proca stars (PSs) [1] - see also [25-27] for generalizations. These configurations have been found in the Einstein-(complex)-Proca system and, in analogy to their scalar cousins, they correspond to a single frequency mode building up a (vector), macroscopic, selfgravitating Bose-Einstein condensate. This frequency appears as a harmonic time dependence for the Proca potential and the domain of existence of PSs is very similar to that of SBSs, when presented in an ADM mass vs Proca frequency diagram-Fig. 1-except that the latter have a lower mass and a wider frequency range.

The stability properties of the spherical PS solutions also parallel those of the spherical SBSs. Analyzing radial perturbations, it was shown in [1] that there is a stable branch of PS solutions, corresponding in Fig. 1 to the line connecting $w / \mu=1$ to the maximal ADM mass, and an

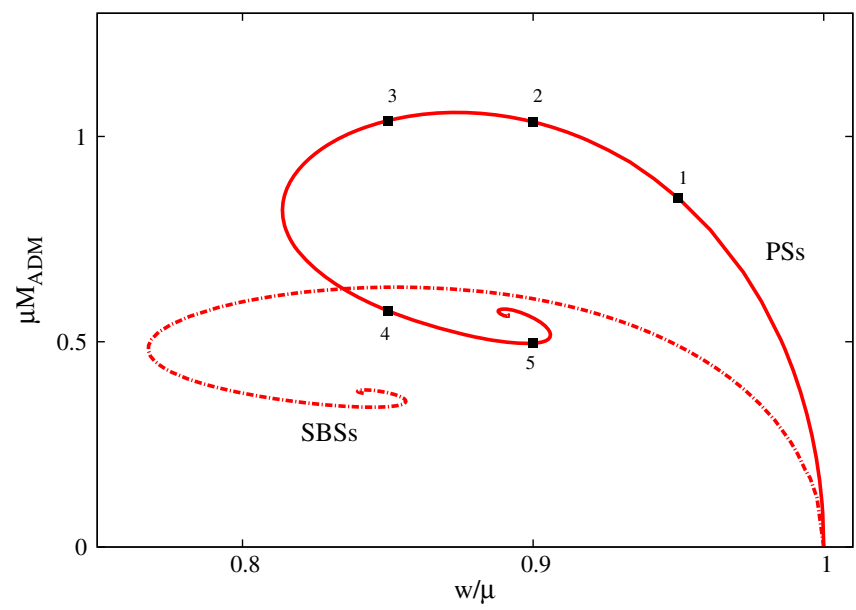

FIG. 1. Domain of existence of the spherical (fundamental) PSs (solid line) and spherical (fundamental) SBSs (dashed line) solutions in an ADM mass vs vector/scalar field frequency diagram. Both fields have been assumed to have mass $\mu$. The five highlighted points correspond to the configurations to be evolved below. unstable branch, corresponding to the remaining part of the spiraling curve. The purpose of this paper is to (1) confirm this picture by performing fully nonlinear time evolutions, rather than a linear frequency domain analysis, and (2) determine the fate of unstable solutions, by evolving a sample of representative cases.

The evolutions described herein confirm the picture obtained from perturbation theory, regarding the existence of a stable branch (connecting the vacuum with the solution with maximal ADM mass) and an unstable branch. For the latter, all configurations we have evolved with no added perturbation (only the discretization error of our numerical code) collapse into a black hole. Introducing a perturbation, however, can change the fate of an unstable solution in a way that depends on the sign of its binding energy. We illustrate this by a particular type of perturbation which makes unstable solutions with positive binding energy migrate to the stable branch, whereas unstable solutions with a negative binding energy (excess energy) undergo fission; i.e. they disperse entirely.

For the cases in which a black hole results from the evolution, after apparent horizon formation, we observe the possibility of a long-lived remnant Proca field lingering outside the horizon. A Fourier analysis, together with a fit of the Proca remnant, suggests this slowly decaying remnant is composed of quasibound states of the Proca field outside a Schwarzschild black hole. This type of wig around a black hole has been observed for the scalar field case in [28], and its lifetime scales with the inverse of the scalar/Proca field mass.

This paper is organized as follows. In Sec. II we present the covariant Einstein-(complex)-Proca model and the equations that are used for the numerical evolutions. In Sec. III we describe the initial data and in particular a set of five representative PS solutions that is used in our

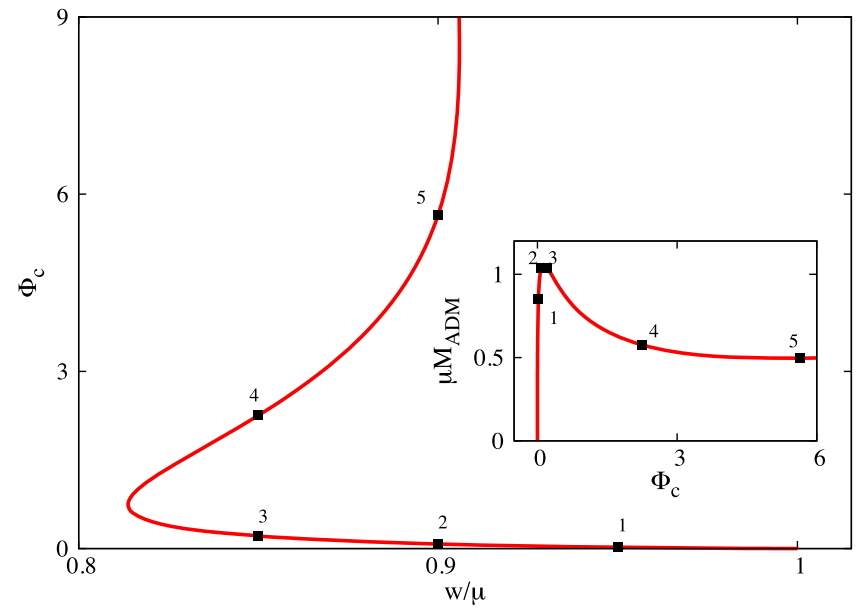

FIG. 2. Magnitude of the Proca electric potential at the origin, $\Phi_{c}(r=0)$, vs the frequency. The five highlighted points correspond to the same as in Fig. 1. The inset shows the ADM mass as a function of $\Phi_{c}(r=0)$. 
numerical evolutions. A brief description of the numerical techniques is given in Sec. IV and our results are presented in Sec. V. Final remarks are presented in Sec. VI. A brief overview of technical details concerning the static Proca star solutions is given in Appendix $\mathrm{A}$ and a succinct assessment of our numerical code is given in Appendix B.

\section{BASIC EQUATIONS}

We investigate the dynamics of a complex Proca field by solving numerically the Proca equations coupled to the Einstein equations. The system is described by the action $\mathcal{S}=\int d^{4} x \sqrt{-g} \mathcal{L}$, where the Lagrangian density depends on the Proca potential $\mathcal{A}$, and field strength $\mathcal{F}=d \mathcal{A}$; it is given by

$$
\mathcal{L}=\frac{R}{16 \pi G_{0}}-\frac{1}{4} \mathcal{F}_{\alpha \beta} \overline{\mathcal{F}}^{\alpha \beta}-\frac{1}{2} \mu^{2} \mathcal{A}_{\alpha} \overline{\mathcal{A}}^{\alpha},
$$

where the bar denotes complex conjugation. In the following we briefly describe the main equations used for the numerical implementation, in order to perform time evolutions.

\section{A. The BSSN equations}

The evolution of the Einstein-Proca system is performed using the numerical code in spherical polar coordinates described in [29], which has been adapted to account for the Proca field (here we set $c=G_{0}=1$ ). We adopt Brown's covariant form [30,31] of the Baumgarte-Shapiro-ShibataNakamura (BSSN) formulation [32-34]. The conformally related spatial metric $\bar{\gamma}_{i j}$ is written as

$$
\bar{\gamma}_{i j}=e^{-4 \phi} \gamma_{i j},
$$

where $\gamma_{i j}$ is the physical spatial metric, and $e^{\phi}$ a conformal factor. We note that

$$
e^{4 \phi}=(\bar{\gamma} / \gamma)^{1 / 3},
$$

with $\gamma=\operatorname{det} \gamma_{i j}$ and $\bar{\gamma}=\operatorname{det} \bar{\gamma}_{i j}$.

We introduce a background connection $\hat{\Gamma}_{j k}^{i}$ and define

$$
\Delta \Gamma_{j k}^{i}=\bar{\Gamma}_{j k}^{i}-\hat{\Gamma}_{j k}^{i}
$$

which, unlike the two connections themselves, transform as a tensor field. We also define the trace of these variables as

$$
\Delta \Gamma^{i} \equiv \bar{\gamma}^{j k} \Delta \Gamma_{j k}^{i} .
$$

It is not necessary for the background connection to be associated with any metric, but in practice we take it to be the Levi-Civita connection of flat spacetime in spherical coordinates.

We define the connection vector $\bar{\Lambda}^{i}$ as a new set of independent variables that are equal to the $\Delta \Gamma^{i}$ when the constraint

$$
\mathcal{C}^{i} \equiv \bar{\Lambda}^{i}-\Delta \Gamma^{i}=0
$$

holds. Defining $\partial_{\perp_{n}} \equiv \partial_{t}-\mathcal{L}_{\beta}$, where $\mathcal{L}_{\beta}$ denotes the Lie derivative along the shift vector $\beta^{i}$, we then obtain the following set of evolution equations:

$$
\begin{aligned}
\partial_{\perp_{n}} \bar{\gamma}_{i j}= & -\frac{2}{3} \bar{\gamma}_{i j} \bar{D}_{k} \beta^{k}-2 \alpha \bar{A}_{i j}, \\
\partial_{\perp_{n}} \bar{A}_{i j}= & -\frac{2}{3} \bar{A}_{i j} \bar{D}_{k} \beta^{k}-2 \alpha \bar{A}_{i k} \bar{A}_{j}^{k}+\alpha \bar{A}_{i j} K \\
& +e^{-4 \phi}\left[-2 \alpha \bar{D}_{i} \bar{D}_{j} \phi+4 \alpha \bar{D}_{i} \phi \bar{D}_{j} \phi\right. \\
& +4 \bar{D}_{(i} \alpha \bar{D}_{j)} \phi-\bar{D}_{i} \bar{D}_{j} \alpha \\
& \left.+\alpha\left(\bar{R}_{i j}-8 \pi S_{i j}\right)\right]^{\mathrm{TF}}, \\
\partial_{\perp_{n}} \phi= & \frac{1}{6} \bar{D}_{k} \beta^{i}-\frac{1}{6} \alpha K, \\
\partial_{\perp_{n}} K= & \frac{\alpha}{3} K^{2}+\alpha \bar{A}_{i j} \bar{A}^{i j}-e^{-4 \phi}\left(\bar{D}^{2} \alpha+2 \bar{D}^{i} \alpha \bar{D}_{i} \phi\right) \\
& +4 \pi \alpha(\rho+S),
\end{aligned}
$$

$$
\begin{aligned}
\partial_{\perp_{n}} \bar{\Lambda}^{i}= & \bar{\gamma}^{j k} \hat{\mathcal{D}}_{j} \hat{\mathcal{D}}_{k} \beta^{i}+\frac{2}{3} \Delta \Gamma^{i} \bar{D}_{j} \beta^{j}+\frac{1}{3} \bar{D}^{i} \bar{D}_{j} \beta^{j} \\
& -2 \bar{A}^{j k}\left(\delta^{i}{ }_{j} \partial_{k} \alpha-6 \alpha \delta^{i}{ }_{j} \partial_{k} \phi-\alpha \Delta \Gamma_{j k}^{i}\right) \\
& -\frac{4}{3} \alpha \bar{\gamma}^{i j} \partial_{j} K-16 \pi \alpha \bar{\gamma}^{i j} S_{j},
\end{aligned}
$$

where $\alpha$ is the lapse function, $\hat{\mathcal{D}}_{i}$ denotes a covariant derivative that is built from the background connection $\hat{\Gamma}_{j k}^{i}$ and the superscript TF denotes the trace-free part. The matter sources $\rho, S_{i}, S_{i j}$ and $S$ denote the density, momentum density, stress, and the trace of the stress as observed by a normal observer, respectively, and are defined by

$$
\begin{aligned}
\rho & \equiv n_{a} n_{b} T^{a b}, \\
S_{i} & \equiv-\gamma_{i a} n_{b} T^{a b}, \\
S_{i j} & \equiv \gamma_{i a} \gamma_{j b} T^{a b}, \\
S & \equiv \gamma^{i j} S_{i j} .
\end{aligned}
$$

Here

$$
n_{a}=(-\alpha, 0,0,0)
$$

is the normal one-form on a spatial slice, and $T^{a b}$ is the stressenergy tensor.

We compute the Ricci tensor $\bar{R}_{i j}$ associated with $\bar{\gamma}_{i j}$ from

$$
\begin{aligned}
\bar{R}_{i j}= & -\frac{1}{2} \bar{\gamma}^{k l} \hat{\mathcal{D}}_{k} \hat{\mathcal{D}}_{l} \bar{\gamma}_{i j}+\bar{\gamma}_{k(i} \hat{\mathcal{D}}_{j)} \bar{\Lambda}^{k}+\Delta \Gamma^{k} \Delta \Gamma_{(i j) k} \\
& +\bar{\gamma}^{k l}\left(2 \Delta \Gamma_{k(i}^{m} \Delta \Gamma_{j) m l}+\Delta \Gamma_{i k}^{m} \Delta \Gamma_{m j l}\right) .
\end{aligned}
$$


The Hamiltonian constraint takes the form

$$
\begin{aligned}
\mathcal{H} \equiv & \frac{2}{3} K^{2}-\bar{A}_{i j} \bar{A}^{i j}+e^{-4 \phi}\left(\bar{R}-8 \bar{D}^{i} \phi \bar{D}_{i} \phi-8 \bar{D}^{2} \phi\right) \\
& -16 \pi \rho \\
= & 0
\end{aligned}
$$

and the momentum constraints can be written as

$$
\begin{aligned}
\mathcal{M}^{i} \equiv & e^{-4 \phi}\left(\frac{1}{\sqrt{\bar{\gamma}}} \hat{\mathcal{D}}_{j}\left(\sqrt{\bar{\gamma}} \bar{A}^{i j}\right)+6 \bar{A}^{i j} \partial_{j} \phi\right. \\
& \left.-\frac{2}{3} \bar{\gamma}^{i j} \partial_{j} K+\bar{A}^{j k} \Delta \Gamma_{j k}^{i}\right)-8 \pi S^{i} \\
= & 0 .
\end{aligned}
$$

\section{B. The Proca equations}

The Proca field is split into its scalar $\Phi, 3$-vector $a_{i}$ potential and a three-dimensional "electric" $\mathbf{E}$ and "magnetic" B fields. The evolution equations for the complex Proca field take the form [35]

$$
\begin{aligned}
\partial_{t} a_{i}= & -\alpha\left(E_{i}+D_{i} \Phi\right)-\Phi D_{i} \alpha+\mathcal{L}_{\beta} a_{i}, \\
\partial_{t} E^{i}= & \alpha\left(K E^{i}+\mu^{2} a^{i}+\epsilon^{i j k} D_{j} B_{k}\right) \\
& -\epsilon^{i j k} B_{j} D_{k} \alpha+\mathcal{L}_{\beta} E^{i}, \\
\partial_{t} B^{i}= & \alpha\left(K B^{i}-\epsilon^{i j k} D_{j} E_{k}\right) \\
& +\epsilon^{i j k} E_{j} D_{k} \alpha+\mathcal{L}_{\beta} B^{i}, \\
\partial_{t} \Phi= & -a^{i} D_{i} \alpha+\alpha\left(K \Phi-D_{i} a^{i}\right)+\mathcal{L}_{\beta} \Phi .
\end{aligned}
$$

The stress-energy tensor of the Proca field reads [1]

$$
\begin{aligned}
T_{a b}= & -\mathcal{F}_{c(a} \overline{\mathcal{F}}_{b)}^{c}-\frac{1}{4} g_{a b} \mathcal{F}_{c d} \overline{\mathcal{F}}^{c d} \\
& +\mu^{2}\left[\mathcal{A}_{(a} \overline{\mathcal{A}}_{b)}-\frac{1}{2} g_{a b} \mathcal{A}_{c} \overline{\mathcal{A}}^{c}\right],
\end{aligned}
$$

from which we can compute the source terms of the Einstein equations. These are given by

$$
\begin{gathered}
8 \pi \rho=\gamma_{i j}\left(E^{i} E^{j}+B^{i} B^{j}\right)+\mu^{2}\left(\Phi^{2}+\gamma^{k l} a_{i} a_{j}\right) \\
4 \pi(\rho+S)=\gamma_{i j}\left(E^{i} E^{j}+B^{i} B^{j}\right)+2 \mu^{2} \Phi^{2}, \\
4 \pi\left(S_{i j}-\frac{S}{3} \gamma_{i j}\right)=-\gamma_{i k} \gamma_{j l}\left(E^{k} E^{l}+B^{k} B^{l}\right) \\
+\frac{\gamma_{i j}}{3}\left(E^{2}+B^{2}\right)+\mu^{2} a_{i} a_{j}-\mu^{2} \frac{\gamma_{i j}}{3} \gamma^{k l} a_{k} a_{l},
\end{gathered}
$$

$$
4 \pi j^{i}=\gamma^{l i} \epsilon_{l j k} E^{j} B^{k}+\mu^{2} \Phi \gamma^{i k} a_{k} .
$$

We can define the "Gauss" constraint as

$$
G=D_{i} E^{i}+\mu^{2} \Phi=0 .
$$

Since for the evolutions reported in this paper we are assuming spherical symmetry, we only need to consider the radial component of the vectors and the magnetic field $\mathbf{B}=0$.

\section{PROCA STAR SOLUTION AND INITIAL DATA}

Proca stars were obtained in [1] as stationary solutions to the model described by the action (1). Here we focus on spherically symmetric Proca stars that we now briefly review, and that are taken as the initial data for the time evolutions performed in the next section.

\section{A. The stationary solutions}

We consider a spherically symmetric line element

$d s^{2}=-e^{2 F_{0}} d t^{2}+e^{2 F_{1}}\left[d r^{2}+r^{2}\left(d \theta^{2}+\sin ^{2} \theta d \varphi^{2}\right)\right]$,

where $F_{0}, F_{1}$ are radial functions and $r, \theta, \varphi$ correspond to isotropic coordinates. The Proca field ansatz is given in terms of another two real functions $\left(V, H_{1}\right)$ which depend also on $r$,

$$
\mathcal{A}=e^{-i w t}\left(i V d t+\frac{H_{1}}{r} d r\right),
$$

where $w>0$ is the frequency of the field. The EinsteinProca equations are solved with appropriate boundary conditions, which are compatible with an approximate construction of the solutions on the boundary of the domain of integration. This ansatz corresponds to the conventions in [1] for the axisymmetric case, specialized to spherical symmetry. Illustrative examples of the four radial functions above, for two spherical Proca star solutions, can be found in Fig. 2 of Ref. [1] (but in a different radial coordinate). Some details on the construction of static Proca star solutions are given in Appendix A.

The magnitude of the Proca electric potential at the origin vs Proca field frequency diagram of the solutions is shown in Fig. 2, where we highlight the sample of numerical solutions that is used in the evolutions reported in Sec. V. The corresponding frequency, ADM mass, Noether charge $Q$ [cf. Eq. (25)], magnitude of the Proca electric potential at the origin [cf. Eq. (26)] and also the apparent horizon $(\mathrm{AH})$ mass resulting from the unperturbed evolution (see the next section), are summarized in Table I. The difference between the ADM mass and the AH mass is due to the numerical error (less than 1\%) that comes mostly from the logarithmic radial grid. Computing the Komar mass with our evolution code gives the same AH mass. 
TABLE I. Spherically symmetric Proca star models.

\begin{tabular}{lccccc}
\hline \hline Model & $\omega / \mu$ & $\mu M_{\mathrm{ADM}}$ & $\mu^{2} Q$ & $\Phi_{c}(r=0)$ & $\mu M_{\mathrm{AH}}$ \\
\hline PS1 & 0.95 & 0.849 & 0.864 & 0.0214 & - \\
PS2 & 0.90 & 1.036 & 1.063 & 0.0779 & - \\
PS3 & 0.85 & 1.039 & 1.065 & 0.2121 & 1.045 \\
PS4 & 0.85 & 0.576 & 0.504 & 2.2547 & 0.578 \\
PS5 & 0.90 & 0.496 & 0.412 & 5.6473 & 0.489 \\
\hline \hline
\end{tabular}

Taking more radial grid points, both converge to the correct Proca mass.

Proca stars carry a conserved Noether charge, $Q$, which is a measure of the number of Proca particles in the BoseEinstein condensate. It is given by integrating the temporal component of the conserved current associated to the global $U(1)$ symmetry on a spacelike surface,

$$
Q=\frac{i}{2} \int_{\Sigma} d^{3} x \sqrt{-g}\left(\overline{\mathcal{F}}^{t \beta} \mathcal{A}_{\beta}-\mathcal{F}^{t \beta} \overline{\mathcal{A}}_{\beta}\right) .
$$

Multiplying this number by the mass of one individual Proca particle, $\mu$, one obtains a measure of the total energy excluding binding energy. A comparison with the ADM mass of each solution then discriminates solutions with a positive binding energy, $M_{\mathrm{ADM}}<\mu Q$, from the ones with a negative binding energy, or excess energy, $M_{\mathrm{ADM}}>\mu Q$. The latter, naturally, only occur in the unstable branch. The separation point (zero binding energy) turns out to occur very close to (but not exactly at) the solution with the minimum frequency (see Fig. 1 in [1]). This means that the classification of our five illustrative solutions is, from perturbation theory plus binding energy considerations, as follows:

(i) Models 1 and 2: stable (which implies positive binding energy);

(ii) Model 3: unstable with positive binding energy;

(iii) Model 4 and 5: unstable with negative binding energy/excess energy.

The translation between the four radial functions above, $F_{0}, F_{1}, V, H_{1}$, and the initial value for the Proca field variables described in Eqs (13)-(16) is given as follows:

$$
\begin{aligned}
\Phi & =-n^{\mu} \mathcal{A}_{\mu}, \\
a_{i} & =\gamma_{i}^{\mu} \mathcal{A}_{\mu}, \\
E^{i} & =-i \frac{\gamma^{i j}}{\alpha}\left(D_{i}(\alpha \Phi)+\partial_{t} a_{j}\right) .
\end{aligned}
$$

Then, from (24), we obtain

$$
\begin{aligned}
& \Phi=-i \frac{V}{\alpha}, \\
& a_{r}=\frac{H_{1}}{r},
\end{aligned}
$$

$$
E^{r}=i \frac{\gamma^{r r}}{\alpha}\left(D_{r} V+\omega \frac{H_{1}}{r}\right) .
$$

In the evolutions to be discussed below, one of the observables of interest is the energy in the Proca field as well as the energy in the black hole, in case one forms. A natural way to assign well-defined such energies in a stationary, asymptotically flat spacetime is by using Komar integrals. The Komar mass at infinity coincides with the ADM mass, $M_{\mathrm{ADM}}$. By using Gauss's law, this mass can be computed as a spatial volume integral of the "Komar energy density" along a spacelike slice from a horizon, in case one exists, up to spatial infinity, plus the horizon contribution, $M_{\mathrm{AH}}$, which is, again, computed as a Komar integral, $M_{\mathrm{ADM}}=M_{\mathrm{AH}}+E_{\mathrm{PF}}$, where [25]

$$
E_{\mathrm{PF}}=-\int_{\Sigma} d r d \theta d \varphi\left(2 T_{t}^{t}-T_{\alpha}^{\alpha}\right) \alpha \sqrt{\gamma} .
$$

In our evolutions we use $E_{\mathrm{PF}}$ with the volume integral computed from the $\mathrm{AH}$ (origin) to spatial infinity, in case an $\mathrm{AH}$ is present (absent). For the horizon mass we use the irreducible mass computed from the horizon area, since the black hole that forms has no electric charge or rotation.

\section{NUMERICS}

As mentioned before, the numerical relativity code we use to perform the evolution of the Einstein-Proca system is based on the code originally presented in [29]. The most salient feature of this code, as compared to other numerical relativity codes, is the fact that the equations are implemented and solved using spherical polar coordinates. The time update of the different systems of evolution equations (Einstein and Proca) is therefore done using the same type of techniques we have extensively used in our previous work. The interested reader is addressed in particular to Refs. [36-38] for complete details.

As a summary, we indicate here that the evolution equations are integrated using the second-order partially implicit Runge-Kutta (PIRK) method developed by $[39,40]$. This method allows us to handle the singular terms that appear in the evolution equations due to our choice of curvilinear coordinates. For the simulations presented in this paper, the original code had to be upgraded to account for the Proca field. Therefore, the evolution scheme for this field is the only feature which is new with respect to previous versions of the code. Therefore, it is worth discussing in some more detail our specific implementation.

Following $[39,40]$ we cast the Einstein-Proca system of partial differential equations as

$$
\left\{\begin{array}{l}
u_{t}=\mathcal{L}_{1}(u, v), \\
v_{t}=\mathcal{L}_{2}(u)+\mathcal{L}_{3}(u, v),
\end{array}\right.
$$


where $\mathcal{L}_{1}, \mathcal{L}_{2}$ and $\mathcal{L}_{3}$ represent general nonlinear differential operators. In the second-order PIRK method the time update from time $t^{n}$ to $t^{n+1}$ is done according to the following two-step algorithm:

$$
\begin{gathered}
\left\{\begin{array}{l}
u^{(1)}=u^{n}+\Delta t L_{1}\left(u^{n}, v^{n}\right), \\
v^{(1)}=v^{n}+\Delta t\left[\frac{1}{2} L_{2}\left(u^{n}\right)+\frac{1}{2} L_{2}\left(u^{(1)}\right)+L_{3}\left(u^{n}, v^{n}\right)\right],
\end{array}\right. \\
\left\{\begin{array}{l}
u^{n+1}=\frac{1}{2}\left[u^{n}+u^{(1)}+\Delta t L_{1}\left(u^{(1)}, v^{(1)}\right)\right], \\
v^{n+1}=v^{n}+\frac{\Delta t}{2}\left[L_{2}\left(u^{n}\right)+L_{2}\left(u^{n+1}\right)+L_{3}\left(u^{n}, v^{n}\right)+L_{3}\left(u^{(1)}, v^{(1)}\right)\right],
\end{array}\right.
\end{gathered}
$$

where $L_{1}, L_{2}$ and $L_{3}$ are the corresponding discrete operators. The explicit form of these operators for the (BSSN) Einstein evolution equations is shown in [29]. Regarding the Proca field, each time step starts by evolving explicitly the vector potential $a_{i}$, i.e. all the source terms of the evolution equations of these variables are included in the $L_{1}$ operator.

We then evolve the scalar potential $\Phi$. More precisely, the corresponding $L_{2}$ and $L_{3}$ operators associated with the evolution equations for $a_{i}$ are

$$
\begin{aligned}
& L_{2(\Phi)}=-a^{i} D_{i} \alpha-\alpha D_{i} a^{i}, \\
& L_{3(\Phi)}=\alpha K \Phi+\mathcal{L}_{\beta} \Phi .
\end{aligned}
$$

The components of the electric field $E^{i}$ are evolved partially implicitly, using the updated values of $\Phi$ and $a_{i}$. The corresponding operators are

$$
\begin{aligned}
L_{2\left(E^{i}\right)}= & \alpha\left(K E^{i}+\mu^{2} a^{i}+\epsilon^{i j k} D_{j} B_{k}\right) \\
& -\epsilon^{i j k} B_{j} D_{k} \alpha, \\
L_{3\left(E^{i}\right)}= & \mathcal{L}_{\beta} E^{i} .
\end{aligned}
$$

Finally, we note that the spatial derivatives in the spacetime evolution are computed using a fourth-order, centered, finite-difference approximation on a logarithmic grid (see [38]). Moreover, as customary in grid-based numerical relativity codes, we use fourth-order KreissOliger dissipation to avoid high frequency noise appearing near the outer boundary. Details about the convergence properties and accuracy of the code are given in Appendix B.

In the numerical simulations we have used a logarithmic radial grid that extends from the origin to $r=50$ and uses a minimum resolution close to the origin of $\Delta r=0.0125$.

\section{RESULTS}

\section{A. Unperturbed evolutions}

We start by evolving the five spherically symmetric Proca star models described in Table I without any additional perturbation (apart from the unavoidable discretization error).
In Fig. 3 we plot the time evolution of the Proca field energy computed from Eq. (32) and the irreducible mass of the $\mathrm{AH}$, when the latter is present. The figure shows that the energy remains constant for models 1 and 2 , hence showing their stability, at least in the absence of larger perturbations. For the other three models, the discretization error is sufficient to trigger the collapse of the solutions. At some point in the evolution an AH forms. This is confirmed in Fig. 4, where the time evolution of the minimum of the lapse function is plotted, showing it tends to 0 , a signature of AH formation. The precise instants those horizons are found in our code are indicated by the three vertical dashed lines in the figure. Subsequently, all Proca field energy evolves towards being absorbed by the black hole. The results in Figs. 3 and 4 also indicate that decay is progressively faster as we move away from the threshold of stability solution (maximal ADM mass) in Fig. 1. These results match precisely the expectation from perturbation theory in the frequency domain [1].

Let us now turn our attention to Fig. 5. This figure shows the time evolution of the amplitude of the scalar potential at some extraction radius and for three representative models. The top panel (model 1) shows constant amplitude oscillations, with a frequency matching that of the Proca stars, as expected for a solution that is not changing in time. The

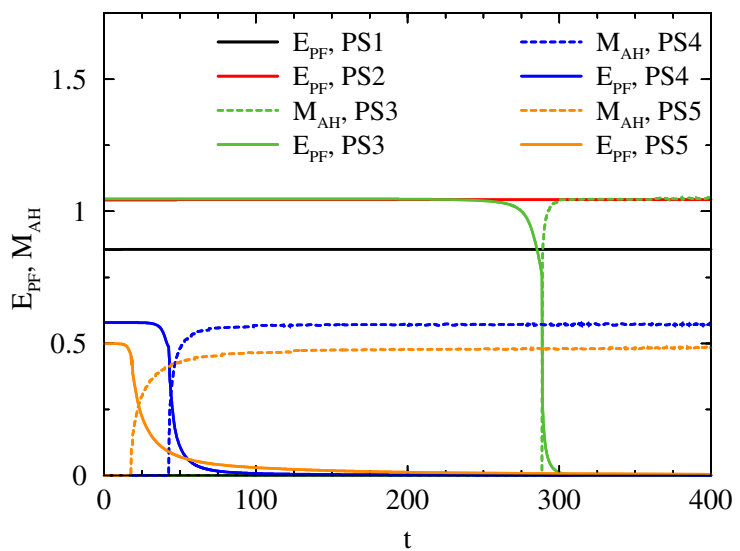

FIG. 3. Time evolution of the Proca field energy and the apparent horizon mass for the unperturbed models 1-5. In this and remaining time series, the time coordinate is given in units of $\mu$. 


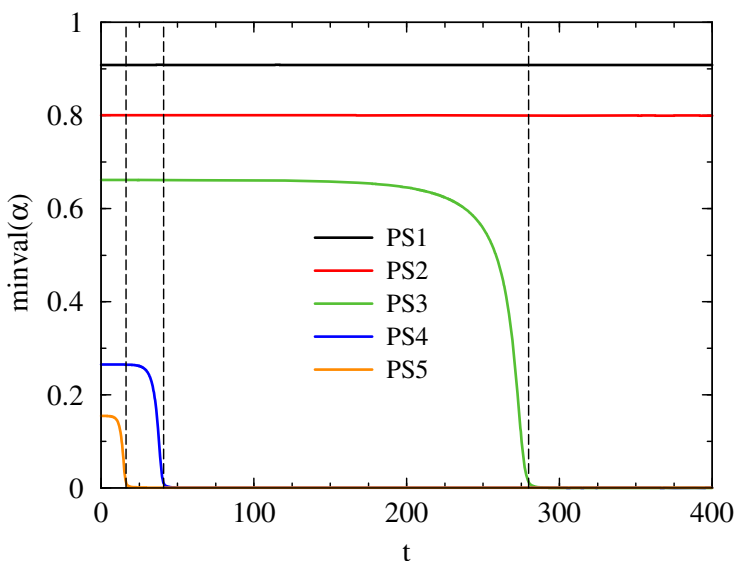

FIG. 4. Time evolution of the minimal value of the lapse for all unperturbed models. The vertical dashed lines indicate the time of formation of an $\mathrm{AH}$ for models 3-5.

Fourier transform of the time series reveals (inset) that the corresponding frequency is that of the Proca star. The middle panel (model 3) shows oscillations that remain constant up to $t \sim 150$, then grow and then decay to 0 . At about $t \sim 300$ the $\mathrm{AH}$ is formed (Fig. 4) and shortly afterwards no noticeable Proca field remains outside the horizon. Again, the inset shows that the frequency in the time series is the one of the original Proca star. The bottom panel (model 5 and likewise for model 4) shows a different behavior from model 3 (middle panel), in spite of both leading to black hole formation. When the black hole is formed, a more significant part of the Proca field lingers for a longer period of time. This is already visible comparing the Proca field energy curves for models 4 or 5 and model 3, in Fig. 3. Moreover, the beating pattern observed in the bottom panel of Fig. 5 shows that more than one significant frequency is present. This is indeed confirmed by a Fourier analysis (inset). Such an analysis can be compared with the frequencies of the first few monopole quasibound states of a Proca field on a Schwarzschild BH [41] — Table II $^{1}$ —and yields the following conclusions:

(i) The initial part of the time series $(t=0-200$, black solid line in the inset) contains the initial Proca star frequency $w / \mu=0.9$; its frequency range is centered around $w / \mu=0.96-0.97$, which matches the fundamental mode frequency, whose real part is $w \sim 0.966-0.970$, for $\mu M_{\mathrm{ADM}}=0.5$ or 0.45 respectively (Table II). The peak frequency is slightly

\footnotetext{
${ }^{1}$ We display the frequencies for both the fundamental mode $(n=0)$ and first overtones $(n=1,2,3)$, where $n$ is the excitation quantum number [41] and for both $\mu M_{\mathrm{ADM}}=0.5$ and $\mu M_{\mathrm{ADM}}=0.45$. Since the original Proca star has mass $\mu M_{\mathrm{ADM}}=0.496$, the mass of the Schwarzschild black hole resulting from the collapse of model 5, around which a small Proca field remnant lingers, is estimated to be in between these two values. We thank J. G. Rosa for providing us with these frequencies.
}
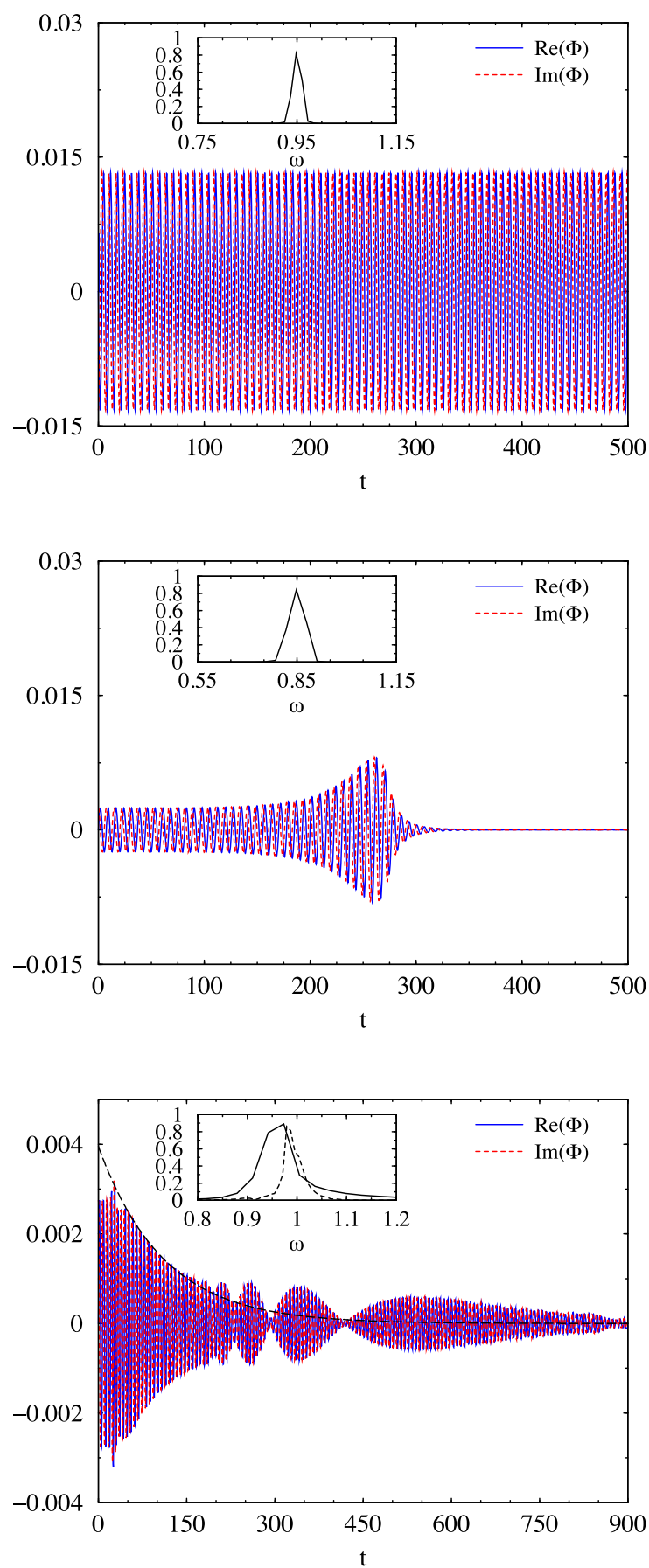

FIG. 5. Time evolutions of the amplitude of the scalar potential extracted at $r=5$ for models 1 (top panel), 3 (middle panel), and 5 (bottom panel). The oscillation frequencies shown in the insets of the top and middle panel are in good agreement with the corresponding $\omega$, in units of $\mu$, of models 1-3 (see Table I). In the bottom panel, the initial decay of the wig is fitted by the enveloping function $e^{-\omega_{I} t}$, where for model $5, \omega_{I} / \mu \sim 0.009$.

higher than the central one, suggesting that an overtone (rather than the fundamental mode) dominates the signal. This is consistent with the enveloping (dashed black) fit curve presented which has 
TABLE II. Frequencies for spherical (monopole) quasibound states of a Proca field (mass $\mu$ ) around a Schwarzschild black hole (mass $M_{\mathrm{ADM}}$ ).

\begin{tabular}{lcc}
\hline \hline Mode & $w / \mu\left(\mu M_{\mathrm{ADM}}=0.45\right)$ & $w / \mu\left(\mu M_{\mathrm{ADM}}=0.5\right)$ \\
\hline$n=0$ & $0.970-0.018 i$ & $0.966-0.025 i$ \\
$n=1$ & $0.986-0.005 i$ & $0.984-0.008 i$ \\
$n=2$ & $0.992-0.002 i$ & $0.991-0.003 i$ \\
$n=3$ & $0.995-0.001 i$ & $0.994-0.002 i$ \\
\hline \hline
\end{tabular}

$\omega_{I} / \mu \sim 0.009$, a value closer to the imaginary part of the first overtone than that of the fundamental mode.

(ii) The later part of the time series $(t=200-900$, dashed line in the inset) is narrower and centered around $w / \mu \sim 0.98-0.99$. The absence of the fundamental mode is expected as its lifetime is $\tau \sim 1 /\left|\omega_{I}\right| \lesssim 100$. The signal is then composed essentially by the overtones which are longer lived than the fundamental mode, and have frequencies in the range $w / \mu \sim 0.98-1$ : Table II. The beating pattern present shows the existence of more than one overtone.

These lingering Proca modes resemble the scalar quasibound states discussed in [28,37] and the beating pattern resembles that observed in superpositions of quasibound states of a scalar field around Schwarzschild black holessee e.g. $[42,43]$.

We recall that test field, frequency domain studies have shown that the Proca field exhibits quasibound states, i.e. solutions which are localized within the vicinity of a black hole [44]. These quasibound states, as in the scalar case, have complex frequencies and the imaginary part corresponds to the decay rate. In the low mass limit, the fundamental modes of these states are consistent with a hydrogenic spectrum. A thorough study of the quasibound states and the quasinormal modes of the Proca field in a Schwarzschild background can be found in [41]. The nonlinear dynamics of self-gravitating Proca quasibound states in both Schwarzschild and Kerr black holes has also been studied numerically in [35]. In particular, the study of Proca fields on the background of rotating black holes has been used to provide upper limits on the mass of the photon [45].

\section{B. Perturbed evolutions}

We turn now to the study of the dynamical behavior of Proca stars under perturbations. To illustrate this situation we add a perturbation (at the few \% level) to the Proca field variables of the form $\tilde{A} \rightarrow \tilde{A} x$ where $\tilde{A}$ stands for $\Phi, a^{r}, E^{r}$ and $x$ is a numerical factor. As a remark, we observe that this type of perturbation does not change the sign of the binding energy of the Proca star. Indeed, inspection of (25) and (32) shows that both these quantities scale as the square of the scaling factor. Thus, this scaling does not change the sign of their difference.
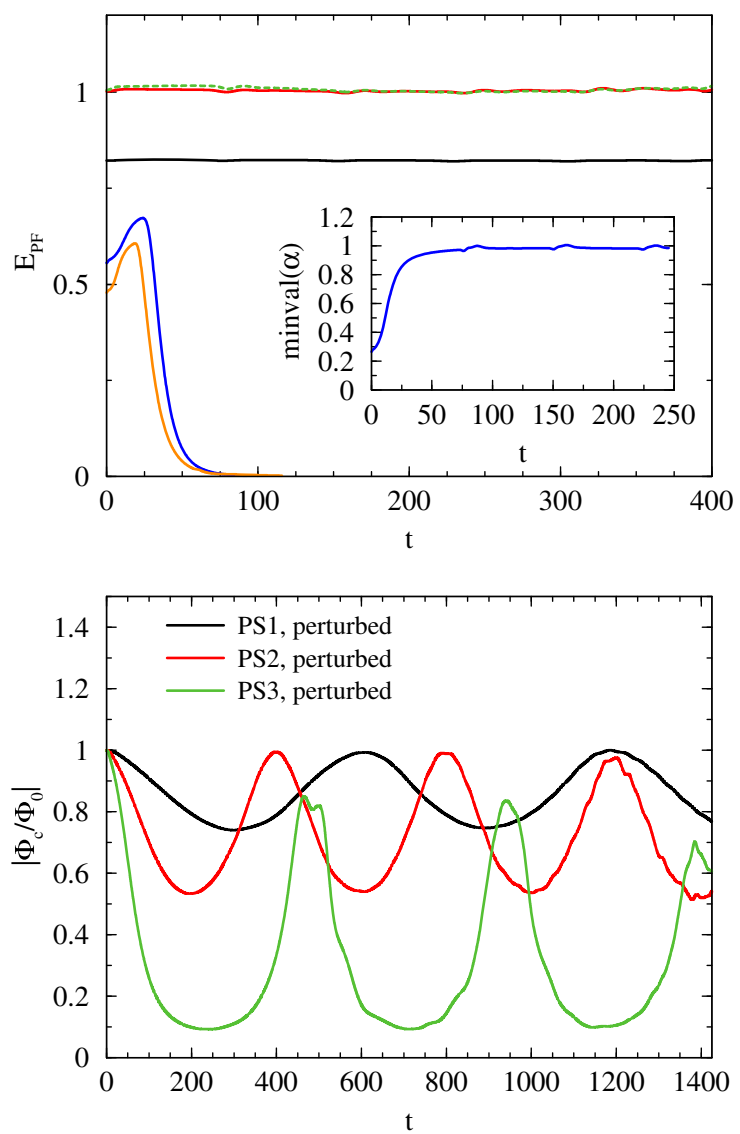

FIG. 6. (Top panel) Time evolution of the perturbed models. The inset shows the minimum value of the lapse of perturbed model 4. (Bottom panel) Time evolution of the magnitude of the Proca electric potential for models 1-3.

We first take $x=1.02$. For this choice, the unstable Proca stars tend to collapse faster than the unperturbed solution with the same resolution. This is the expected result, since this perturbation increases, in particular, the central electric potential of the Proca star (cf. discussion on neutron stars in the conclusions).

Next, we choose $x=0.98$. In this case, we are removing part of the Proca field and the outcome of the evolution is different for the unstable models, as exhibited in Fig. 6. In the top panel of Fig. 6, we plot the time evolution of the Proca field energy for the perturbed models (the color coding is the same as in Fig. 3). Models 1 (black solid line) appears stable. Models 2 (red solid line) and 3 (green dashed line), despite being fairly stable, exhibit some noticeable oscillations. We come back to these shortly. Models 4 and 5, on the other hand, show a total dispersive behavior: the energy of the Proca field goes to 0 without $\mathrm{AH}$ formation; moreover, the value of the lapse function tends to unity at the origin (inset), which is the behavior of flat spacetime. We remark that the slight increase in the Proca field energy clearly visible in the beginning of the evolution of models 4 and 5 is related to the evolution of the lapse function, as it adapts to the perturbation. 
TABLE III. Final state of the five representative PS models considered in this paper.

\begin{tabular}{lcccc}
\hline \hline Model & Perturbative analysis & Binding energy & Evolution (no perturbation) & Evolution $(\times 0.98$ perturbation) \\
\hline PS1 & Stable & $>0$ & Stable & Stable \\
PS2 & Stable & $>0$ & Stable & Stable \\
PS3 & Unstable & $>0$ & Collapse to BH & Migration \\
PS4 & Unstable & $<0$ & Collapse to BH (+noticeable wig) & Dispersion \\
PS5 & Unstable & $<0$ & Collapse to BH (+noticeable wig) & Dispersion \\
\hline \hline
\end{tabular}

A more detailed investigation of what occurs for models 1-3 can be made by analyzing the behavior of the electric potential at the origin, which is displayed in the bottom panel of Fig. 6. All three models oscillate, but whereas the oscillations of models 1 and 2 are not damping the value of $\Phi_{c}$, the ones of model 3 are clearly decreasing its value. We recall, from Fig. 2, that this scalar potential at the origin defines uniquely static Proca solutions. The decrease of its value for model 3 can be interpreted as a migration of the unstable star towards the stable branch. This conclusion is supported by computing the oscillation frequency of the perturbed models. For the perturbed model 3 it is $\sim 0.92$, whereas for the perturbed model 2 , it is $\sim 0.91$. Thus, the perturbed model 3 is oscillating with a frequency which corresponds to a stable Proca star, very close to the one of model 2, which is in the stable branch. We recall that models 4 and 5 have negative binding energy (excess energy), unlike solution 3. It is therefore expectable that they could have a different evolution and disperse away. This is precisely what we observe in these perturbed evolutions.

To summarize, the perturbed evolutions indicate that, depending on the perturbation, but also on the sign of the binding energy, Proca stars can be subject to three different outcomes: collapse, dispersion/fission or migration.

\section{DISCUSSION}

In this paper we have used numerical relativity techniques to study the stability and evolution of Proca stars. These are macroscopic, self-gravitating Bose-Einstein condensates composed by a massive, complex, vector field [1]. Considering the energy of these solutions, together with perturbative stability studies, leads to three different classes of solutions: (i) stable solutions (our models 1 and 2); (ii) unstable solutions with positive binding energy (our model 3); and (iii) unstable solutions with negative binding energy or excess energy (our models 4 and 5). Here, we have shown that the fate of the unstable solutions depends not only on the type of solutions but also on the type of perturbation. Our results are summarized in Table III and as follows.

When we evolve in time the configurations with no perturbations we observe the following behaviors: Models 1 and 2 evolve in time with almost no changes. The Proca field oscillates and the spacetime remains stationary.
Configuration 3 collapses to form a black hole without a noticeable Proca field remnant, thus yielding a vacuum spacetime. For unstable configurations 4 and 5, however, even though a black hole is formed after the collapse a noticeable set of Proca quasibound states remains in its vicinity.

When we apply a perturbation, multiplying the fields by a constant of order unity, the stable configurations still display no evolution. Configuration 3 migrates towards stable states with lower mass, whereas configurations 4 and 5 are no more localized states and the Proca field disperses away.

The fates we have just described for Proca stars parallel quite closely those observed for scalar boson stars and discussed in detail in the introduction. But it is interesting to compare this dynamics with that of the well-known compact fermionic stars, namely neutron stars.

In an astrophysical context, the dynamical fate of neutron stars out of equilibrium has received enormous attention, as it plays a crucial role in significant scenarios of relativistic astrophysics such as supernovae, gamma-ray bursts, or binary neutron star mergers. Moreover, as a fundamental physics problem, critical phenomena in neutron stars at the threshold of black hole formation have also been examined for perfect fluid matter (see [46] and references therein). By triggering the collapse of initially stable models through suitable perturbations, it has been possible to elucidate the boundary between different types of critical behavior in neutron stars and its relationship with the boundary between dispersed and bound end states [46].

Radially unstable equilibrium models of neutron stars at very high densities can be built for any equation of state. Such models satisfy the condition $\partial M_{\mathrm{ADM}} / \partial \rho_{\mathrm{c}}<0$, where $\rho_{\mathrm{c}}$ is the central rest-mass density. Therefore, any perturbation causes them to either collapse to a black hole or to expand to the stable branch of equilibrium models, at smaller central densities. The sign of the perturbation determines the path the unstable star follows. These observations parallel closely what we have described for bosonic stars (both scalar and vector).

While the two outcomes are mathematically viable, the migrating (i.e. expanding) path is ruled out from an astrophysical viewpoint. In a realistic scenario, stable neutron stars, as those formed following a supernova core collapse or an accretion-induced collapse of a white dwarf or those in x-ray binaries, can accrete matter and secularly 
move towards larger central densities along the stable branch of equilibrium configurations. Beyond the maximum-mass limit collapse to a black hole ensues. There is, however, no secular mechanism that could evolve an unstable star to the stable branch. Similarly, if Proca stars form in nature, one may wonder if the migration dynamics we have observed is realized by any naturally occurring mechanism.

The nonlinear dynamics of a migrating relativistic spherical star was first investigated in [47], using a polytropic model with a central rest-mass density larger than that of the maximum-mass stable model. Under the radial perturbation induced by the truncation error of the numerical code, the star was found to expand and evolve towards a stable equilibrium configuration with a smaller central rest-mass density but with approximately the same rest-mass of the perturbed star. The stable configuration is reached asymptotically, as the star oscillates around a central density close to that of a stable star with the same rest mass. A similar behavior has been observed in numerical simulations of relativistic boson stars $[19,22]$. Contrary to the dispersive boson star case, for a neutron star with an ideal-fluid equation of state, the oscillations are gradually damped due to the dissipation of kinetic energy via shock heating.

We close with two remarks. Even though our results suggest that the dynamics and final states of PS is quite similar to SBS, the study of vector fields may yield extra value as compared to the scalar case. As a first example, consider the dynamics of Proca fields around black holes, which has already been considered in the literature, albeit less studied than the corresponding scalar dynamics. The existence of long-lived configurations around nonrotating black holes has been demonstrated in the test field limit $[41,44]$ and in the full nonlinear regime solving the Einstein-Proca equations [35]. The inherent difficulty of adding rotation to the black hole is also present and a full evolution of a rotating black hole with a Proca field is not yet available. Still, since the super-radiant instability of a Proca field has a shorter time scale than its scalar counterpart [48], this offers a promising model for analyzing the nonlinear development of the super-radiant instability around Kerr black holes. Secondly, it has been recently argued [49] that photons can behave near stellar mass black holes as Proca particles, in view of the effective mass induced by the galactic plasma. Thus, the study of real Proca field interacting with black holes may describe astrophysical processes without invoking new exotic particles.

\section{ACKNOWLEDGMENTS}

This work has been supported by the Spanish Ministerio de Economía, Industria y Competitividad (MINECO) (Grants No. AYA2013-40979-P and No. AYA201566899-C2-1-P), by the Generalitat Valenciana (Grants
No. PROMETEOII-2014-069 and No. ACIF/2015/216), by the Consejo Nacional de Ciencia y Tecnología (CONACyT)-México, by the Fundação para a Ciência e a Tecnologia (FCT) (Portugal) IF program, by the Center for Research \& Development in Mathematics and Applications (CIDMA) (FCT) strategic project Grant No. UID/MAT/04106/2013 and by the European Union's Horizon 2020 research and innovation program under the Marie Sklodowska-Curie Grant No. 690904 and by the CIDMA project Grant No. UID/MAT/04106/2013. Computations have been performed at the Servei d'Informàtica de la Universitat de València and at the Blafis cluster at the University of Aveiro.

\section{APPENDIX A: SPHERICALLY SYMMETRIC PROCA STAR SOLUTIONS}

Spherically symmetric Proca stars solutions were discussed in Ref. [1]. In a Schwarschild-like coordinate system the element of line is

$d s^{2}=-N(\bar{r}) \sigma^{2}(\bar{r}) d t^{2}+\frac{d \bar{r}^{2}}{N(\bar{r})}+\bar{r}^{2}\left(d \theta^{2}+\sin ^{2} d \varphi^{2}\right)$.

Comparison of the above line element with the isotropic metric (23) yields for the transformation

$\frac{d r}{r}=\frac{d \bar{r}}{\bar{r} \sqrt{N(\bar{r})}}, \quad$ while $e^{F_{0}}=\sigma \sqrt{N}, \quad e^{F_{1}}=\frac{\bar{r}}{r}$.

For the isotropic coordinates used in this paper, the Einstein equations reduce to two second-order equations for the metric functions $F_{0}, F_{1}$ (reinserting $G_{0}$ ),

$$
\begin{aligned}
& F_{0}^{\prime \prime}+F_{0}^{\prime}\left(\frac{2}{r}+F_{0}^{\prime}+F_{1}^{\prime}\right) \\
& -4 \pi G_{0} e^{-2 F_{0}}\left[\left(V^{\prime}+\frac{w H_{1}}{r}\right)^{2}+2 e^{2 F_{1}} \mu^{2} V^{2}\right]=0, \\
& F_{1}^{\prime \prime}-F_{0}^{\prime} F_{1}^{\prime}+\frac{1}{r}\left(F_{1}^{\prime}-F_{0}^{\prime}\right) \\
& \quad+4 \pi G_{0} \mu^{2}\left(\frac{H_{1}^{2}}{r^{2}}+e^{2\left(F_{1}-F_{0}\right)} V^{2}\right)=0
\end{aligned}
$$

together with the constraint equation

$$
\begin{aligned}
0= & F_{1}^{\prime 2}+2 F_{0}^{\prime} F_{1}^{\prime}+\frac{2}{r}\left(F_{0}^{\prime}+F_{1}^{\prime}\right)+4 \pi G_{0} \\
& \times\left[e^{-2 F_{0}}\left(V^{\prime}+\frac{w H_{1}}{r}\right)^{2}-\mu^{2}\left(\frac{H_{1}^{2}}{r^{2}}+e^{2\left(F_{1}-F_{0}\right)} V^{2}\right)\right] .
\end{aligned}
$$

The Proca equations are

$$
w V^{\prime}+\frac{H_{1}}{r}\left(w^{2}-e^{2 F_{0}} \mu^{2}\right)=0,
$$




$$
\begin{aligned}
V^{\prime \prime} & +\frac{2 V^{\prime}}{r}+\left(V^{\prime}+\frac{w H_{1}}{r}\right)\left(F_{1}^{\prime}-F_{0}^{\prime}\right)+\frac{w}{r^{2}}\left(H_{1}+r H_{1}^{\prime}\right) \\
& -e^{2 F_{1}} \mu^{2} V=0
\end{aligned}
$$

the vector potential being subject to the gauge condition $\nabla_{\alpha} \mathcal{A}^{\alpha}=0$, which is actually a requirement from the field equations for the Proca field

$$
H_{1}^{\prime}+\left(F_{0}^{\prime}+F_{1}^{\prime}+\frac{1}{r}\right) H_{1}-e^{2\left(F_{1}-F_{0}\right)} r w V=0 .
$$

For this ansatz, the Noether charge reads

$$
Q=4 \pi \int_{0}^{\infty} d r e^{F_{1}-F_{0}} H_{1}\left(w H_{1}+r V^{\prime}\right),
$$

and the energy density measured by a static observer is

$$
\begin{aligned}
-T_{t}^{t}= & \frac{1}{2} e^{-2\left(F_{1}+F_{0}\right)}\left(V^{\prime}+\frac{w H_{1}}{r}\right)^{2} \\
& +\frac{1}{2} \mu^{2}\left(\frac{e^{2 F_{1}} H_{1}^{2}}{r^{2}}+e^{-2 F_{0}} V^{2}\right),
\end{aligned}
$$

while the Komar energy density entering the integral (32) is $2 T_{t}^{t}-T_{\alpha}^{\alpha}=-e^{2\left(F_{1}+F_{0}\right)}\left(V^{\prime}+\frac{w H_{1}}{r}\right)^{2}-2 e^{-2 F_{0}} \mu^{2} V^{2}$.

The PSs possess the following expansion at the origin $r \rightarrow 0$,

$$
\begin{aligned}
F_{0}(r) & =f_{0}+\frac{4 \pi G_{0}}{3} e^{2\left(f_{1}-f_{0}\right)} \mu^{2} v_{0}^{2} r^{2}+\mathcal{O}\left(r^{4}\right), \\
F_{1}(r) & =f_{1}-\frac{4 \pi G_{0}}{12} e^{2\left(f_{1}-f_{0}\right)} \mu^{2} v_{0}^{2} r^{2}+\mathcal{O}\left(r^{4}\right), \\
H_{1}(r) & =\frac{1}{3} e^{2\left(f_{1}-f_{0}\right)} v_{0} w r^{2}+\mathcal{O}\left(r^{4}\right), \\
V(r) & =v_{0}+\frac{1}{6} e^{2\left(f_{1}-f_{0}\right)} v_{0}\left(e^{2 f_{0}} \mu^{2}-w^{2}\right) r^{2}+\mathcal{O}\left(r^{4}\right),
\end{aligned}
$$

where $f_{1}, f_{0}$ and $v_{0}$ are constants fixed by the numerics. The first terms in the expression of the solution as $r \rightarrow \infty$ read

$$
\begin{aligned}
& F_{0}(r)=-\frac{M_{\mathrm{ADM}}}{r}+\ldots, \\
& F_{1}(r)=\frac{M_{\mathrm{ADM}}}{r}+\ldots,
\end{aligned}
$$

$$
\begin{gathered}
H_{1}(r)=-c_{0} \frac{w}{\sqrt{\mu^{2}-w^{2}}} e^{-r \sqrt{\mu^{2}-w^{2}}}+\ldots, \\
V(r)=c_{0} \frac{e^{-r \sqrt{\mu^{2}-w^{2}}}}{r}+\ldots,
\end{gathered}
$$

where $c_{0}$ is a constant. Observe that the PSs should satisfy the bound state condition $w<\mu$.

The solutions that smoothly interpolate between the two above asymptotic behaviors are found numerically. In numerical treatment, we set $\mu=1,4 \pi G_{0}=1$, by using a scaled radial coordinate $r \rightarrow r \mu$ (together with $w \rightarrow w / \mu$ ) and scaled potentials $H_{1} \rightarrow H_{1} \sqrt{4 \pi G_{0}}, V \rightarrow V \sqrt{4 \pi G_{0}}$. Restricting to the fundamental set of solutions, we obtain the $(w, M)$-diagram, exhibited in Fig. 1. This spiral starts from $M=0$ for $w / \mu=1$, in which limit the Proca field becomes very diluted and the solution trivializes. At some intermediate frequency $w_{m} / \mu \simeq 0.875$, a maximal ADM mass $M_{\max } \simeq 1.058$ is attained. There is also a minimal allowed frequency $w_{\min } \simeq 0.814$ where $M \sim 0.82$.

\section{APPENDIX B: CODE ASSESSMENT}

Our numerical code was originally developed by [29]. The evolution equations are solved using techniques we have extensively tested and employed in previous works before [36-38]. For the current work, we upgraded the code to account for the Proca field. This appendix hence reports a succinct assessment of the upgraded code. We discuss in particular its convergence properties and the violations of the constraint equations, both for the Einstein and the Proca fields.

Figure 7 shows the convergence properties of the code. This figure displays the time evolution of the rescaled L2 norm of the Hamiltonian constraint (top panel) and of the Gauss constraint (bottom panel) for the unstable model 5. The L2 norm of a constraint $C$ is given by

$$
|C|^{2}=\sqrt{\frac{\sum_{i=1}^{N} C_{i}^{2}}{N}}
$$

where $N$ indicates the number of radial grid points. The results have been obtained at three different radial resolutions, as indicated in the figure caption, and the blue and red solid curves have been multiplied by the appropriate numerical factors, 2 and 4, respectively, corresponding to second-order convergence (thus the three curves should overlap). The constraints are computed in the entire computational domain; hence the black hole puncture of this unstable model is also included. Black hole formation coincides with the rapid initial growth of the constraints. The violation of the 

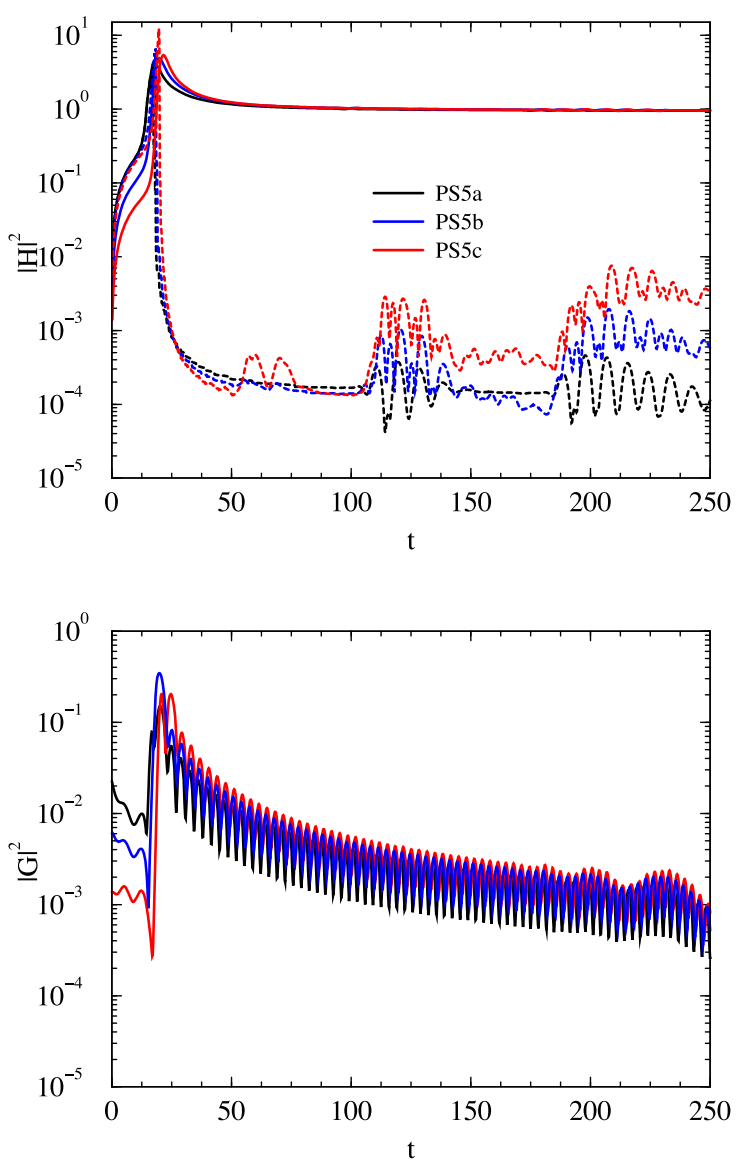

FIG. 7. Convergence analysis for model PS5 employing three different resolutions, $\Delta r=0.0125$, black curves, $\Delta r=$ $0.0125 / \sqrt{2}$, blue curves, and $\Delta r=0.0125 / 2$, red curves. Top panel: L2 norm of the Hamiltonian constraint computed either at all radial points (solid lines) or outside of the $\mathrm{AH}$ (dashed lines). Bottom panel: L2 norm of the Gauss constraint computed at all radial points.

Hamiltonian constraint levels off shortly thereafter and does not subsequently grow. Correspondingly, the violation of the Gauss constraint has decreased by about 2 orders of magnitude by the end of the simulation with respect to the value attained at the moment the black hole forms. In addition, the top panel of Fig. 7 also shows the Hamiltonian constraint when computed outside of the $\mathrm{AH}$ (as customary in numerical relativity studies, see e.g. [50,51]), which is indicated by the dashed lines. In this case, they show a fourth-order convergence, dominated by the spatial derivatives, and are multiplied by 4 and 16 . Observe that the oscillations that appear after $\mathrm{t}=100$ are reflections on the outer boundary, which is at $\mathrm{r}=50$; we have checked, however, that these oscillations do not affect the results by putting the boundary further away. The overall effect of the reflection is to reduce the fourth order of convergence. As expected, when the causally disconnected $\mathrm{AH}$ interior is excluded in the L2 norm computation, the
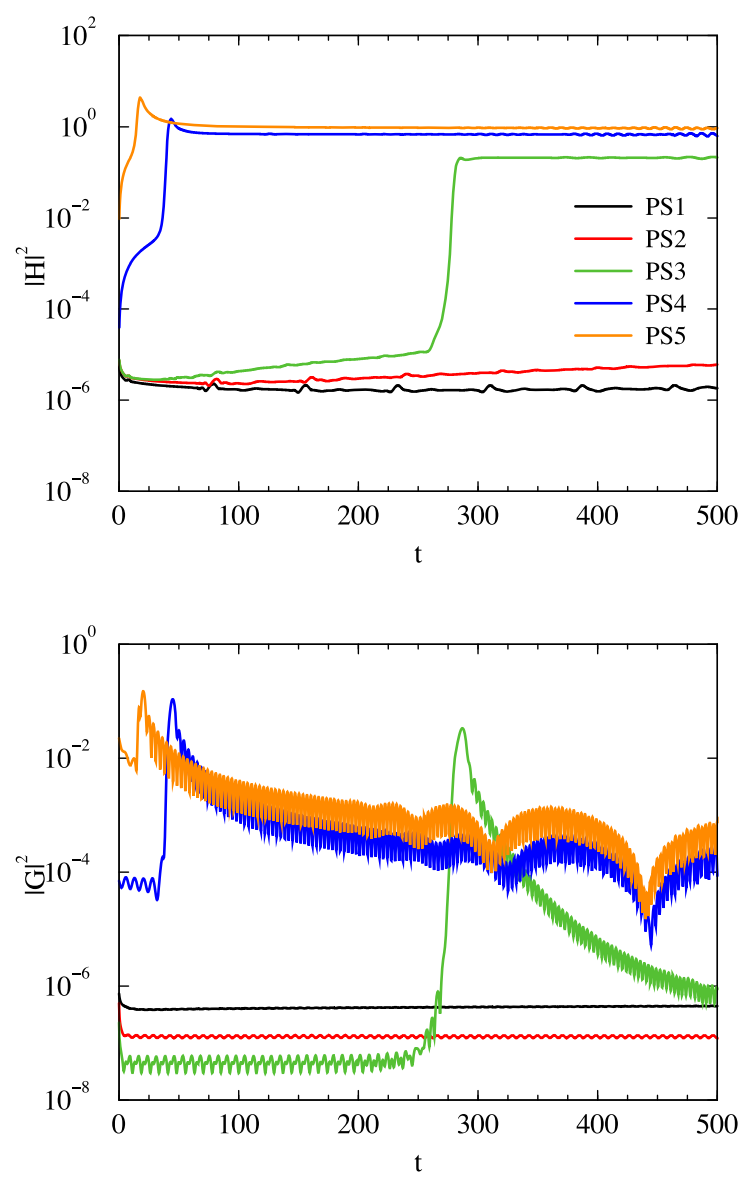

FIG. 8. Time evolution of the L2 norm of the constraints for all Proca star models, using our canonical radial resolution of $\Delta r=0.0125$. Top panel: Hamiltonian constraint. Bottom panel: Gauss constraint.

violations are reduced by several orders of magnitude. All in all, Fig. 7 shows that the PIRK time-evolution scheme of our numerical code has a global order of convergence close to 2 .

Finally, Fig. 8 shows the time evolution of the L2 norm of the Hamiltonian constraint and of the Gauss constraint for all of our Proca star models. Both quantities are computed using all radial grid points, hence including the $\mathrm{AH}$ of the resulting black holes for models $3-5$. The time evolution of the constraints for these models is characterized by the sudden growth at black hole formation, after which the Hamiltonian constraint attains a constant value and the Gauss constraint significantly drops. For the collapsing models, the higher the value of $\Phi_{c}$ the larger the violation of the constraints. On the other hand, for stable models 1 and 2, the violation of the constraints hardly grows in time and it stays at maximum values that are several orders of magnitude smaller than that of unstable models.

It should be noted, however, that the specific maximum violation the constraints attain is somewhat meaningless, as 
this number may be arbitrarily reduced even at the same grid resolution. This can be achieved by taking a larger number of grid points and placing the outer boundary at the appropriate (further out) location, as follows from the definition of the L2 norm in Eq. (B1). Therefore, what is important when assessing the numerical code is to show that the constraints are bounded in time and that the numerical code is convergent.
[1] R. Brito, V. Cardoso, C. A. R. Herdeiro, and E. Radu, Phys. Lett. B 752, 291 (2016).

[2] S. R. Coleman, Nucl. Phys. B262, 263 (1985); B269, 744 (E) (1986)

[3] D. J. Kaup, Phys. Rev. 172, 1331 (1968).

[4] R. Ruffini and S. Bonazzola, Phys. Rev. 187, 1767 (1969).

[5] F. E. Schunck and E. W. Mielke, Classical Quantum Gravity 20, R301 (2003).

[6] G. H. Derrick, J. Math. Phys. (N.Y.) 5, 1252 (1964).

[7] P. Grandclement, G. Fodor, and P. Forgacs, Phys. Rev. D 84, 065037 (2011).

[8] E. Seidel and W. M. Suen, Phys. Rev. Lett. 66, 1659 (1991).

[9] F. E. Schunck and E. W. Mielke, Phys. Lett. A 249, 389 (1998).

[10] S. Yoshida and Y. Eriguchi, Phys. Rev. D 56, 762 (1997).

[11] M. Colpi, S. L. Shapiro, and I. Wasserman, Phys. Rev. Lett. 57, 2485 (1986).

[12] B. Kleihaus, J. Kunz, M. List, and I. Schaffer, Phys. Rev. D 77, 064025 (2008)

[13] F. D. Ryan, Phys. Rev. D 55, 6081 (1997).

[14] P. Grandclement, C. Som, and E. Gourgoulhon, Phys. Rev. D 90, 024068 (2014).

[15] C. A. R. Herdeiro, E. Radu, and H. Rnarsson, Phys. Rev. D 92, 084059 (2015)

[16] S. L. Liebling and C. Palenzuela, Living Rev. Relativ. 15, 6 (2012).

[17] M. Gleiser and R. Watkins, Nucl. Phys. B319, 733 (1989).

[18] T. D. Lee and Y. Pang, Nucl. Phys. B315, 477 (1989).

[19] E. Seidel and W.-M. Suen, Phys. Rev. D 42, 384 (1990).

[20] J. Balakrishna, E. Seidel, and W.-M. Suen, Phys. Rev. D 58, 104004 (1998).

[21] F. S. Guzman, Phys. Rev. D 70, 044033 (2004).

[22] S. H. Hawley and M. W. Choptuik, Phys. Rev. D 62, 104024 (2000).

[23] E. Seidel and W.-M. Suen, Phys. Rev. Lett. 72, 2516 (1994).

[24] A. R. Liddle and M. S. Madsen, Int. J. Mod. Phys. D 01, 101 (1992).

[25] C. Herdeiro, E. Radu, and H. Runarsson, Classical Quantum Gravity 33, 154001 (2016).

[26] F. Garca and I. S. Landea, Phys. Rev. D 94, 104006 (2016).

[27] M. Duarte and R. Brito, Phys. Rev. D 94, 064055 (2016).

[28] J. Barranco, A. Bernal, J. C. Degollado, A. Diez-Tejedor, M. Megevand, M. Alcubierre, D. Núñez, and O. Sarbach, Phys. Rev. Lett. 109, 081102 (2012).
[29] T. W. Baumgarte, P. J. Montero, I. Cordero-Carrión, and E. Müller, Phys. Rev. D 87, 044026 (2013).

[30] J. D. Brown, Phys. Rev. D 79, 104029 (2009).

[31] M. Alcubierre and M. D. Mendez, Gen. Relativ. Gravit. 43, 2769 (2011).

[32] T. Nakamura, K. Oohara, and Y. Kojima, Prog. Theor. Phys. Suppl. 90, 1 (1987).

[33] M. Shibata and T. Nakamura, Phys. Rev. D 52, 5428 (1995).

[34] T. W. Baumgarte and S. L. Shapiro, Phys. Rev. D 59, 024007 (1998).

[35] M. Zilho, H. Witek, and V. Cardoso, Classical Quantum Gravity 32, 234003 (2015).

[36] P. J. Montero and I. Cordero-Carrion, Phys. Rev. D 85, 124037 (2012).

[37] N. Sanchis-Gual, J. C. Degollado, P. J. Montero, and J. A. Font, Phys. Rev. D 91, 043005 (2015).

[38] N. Sanchis-Gual, J. C. Degollado, P. J. Montero, J. A. Font, and V. Mewes, Phys. Rev. D 92, 083001 (2015).

[39] I. Cordero-Carrión and P. Cerdá-Durán, arXiv:1211.5930.

[40] I. Cordero-Carrión and P. Cerdá-Durán, Advances in Differential Equations and Applications, SEMA SIMAI Springer Series Vol. 4 (Springer International Publishing, Switzerland, 2014).

[41] J. G. Rosa and S. R. Dolan, Phys. Rev. D 85, 044043 (2012).

[42] H. Okawa, H. Witek, and V. Cardoso, Phys. Rev. D 89, 104032 (2014).

[43] J. Barranco, A. Bernal, J. C. Degollado, A. Diez-Tejedor, M. Megevand et al., Phys. Rev. D 89, 083006 (2014).

[44] D. V. Gal'tsov, G. V. Pomerantseva, and G. A. Chizhov, Izv. Vuz. Fiz. 27, 81 (1984) [Sov. Phys. J. 27, 697 (1984)].

[45] P. Pani, V. Cardoso, L. Gualtieri, E. Berti, and A. Ishibashi, Phys. Rev. Lett. 109, 131102 (2012).

[46] S. C. Noble and M. W. Choptuik, Phys. Rev. D 93, 024015 (2016).

[47] J. A. Font, T. Goodale, S. Iyer, M. Miller, L. Rezzolla, E. Seidel, N. Stergioulas, W.-M. Suen, and M. Tobias, Phys. Rev. D 65, 084024 (2002).

[48] P. Pani, V. Cardoso, L. Gualtieri, E. Berti, and A. Ishibashi, Phys. Rev. D 86, 104017 (2012).

[49] J. P. Conlon and C. A. R. Herdeiro, arXiv:1701.02034.

[50] D. Alic, W. Kastaun, and L. Rezzolla, Phys. Rev. D 88, 064049 (2013).

[51] C. Reisswig, C. D. Ott, E. Abdikamalov, R. Haas, P. Mösta, and E. Schnetter, Phys. Rev. Lett. 111, 151101 (2013). 